ARTICLE

Received 13 May 2014 | Accepted 4 Sep 2014 | Published 10 Oct $2014 \quad$ DOl: 10.1038/ncomms6154 OPEN

\title{
Visualized effect of oxidation on magnetic recording fidelity in pseudo-single-domain magnetite particles
}

Trevor P. Almeida1, Takeshi Kasama², Adrian R. Muxworthy'1, Wyn Williams³, Lesleis Nagy³, Thomas W. Hansen ${ }^{2}$, Paul D. Brown ${ }^{4} \&$ Rafal E. Dunin-Borkowski ${ }^{5}$

Magnetite $\left(\mathrm{Fe}_{3} \mathrm{O}_{4}\right)$ is an important magnetic mineral to Earth scientists, as it carries the dominant magnetic signature in rocks, and the understanding of its magnetic recording fidelity provides a critical tool in the field of palaeomagnetism. However, reliable interpretation of the recording fidelity of $\mathrm{Fe}_{3} \mathrm{O}_{4}$ particles is greatly diminished over time by progressive oxidation to less magnetic iron oxides, such as maghemite $\left(\gamma-\mathrm{Fe}_{2} \mathrm{O}_{3}\right)$, with consequent alteration of remanent magnetization potentially having important geological significance. Here we use the complementary techniques of environmental transmission electron microscopy and off-axis electron holography to induce and visualize the effects of oxidation on the magnetization of individual nanoscale $\mathrm{Fe}_{3} \mathrm{O}_{4}$ particles as they transform towards $\gamma-\mathrm{Fe}_{2} \mathrm{O}_{3}$. Magnetic induction maps demonstrate a change in both strength and direction of remanent magnetization within $\mathrm{Fe}_{3} \mathrm{O}_{4}$ particles in the size range dominant in rocks, confirming that oxidation can modify the original stored magnetic information.

\footnotetext{
${ }^{1}$ Department of Earth Science and Engineering, Imperial College London, South Kensington Campus, London SW7 2AZ, UK. ${ }^{2}$ Center for Electron Nanoscopy, Technical University of Denmark, Kongens Lyngby DK-2800, Denmark. ${ }^{3}$ School of GeoSciences, University of Edinburgh, The King's Buildings, West Mains Road, Edinburgh EH9 3JW, UK. ${ }^{4}$ Division of Materials, Mechanics and Structures, Department of Mechanical, Materials and Manufacturing Engineering, Faculty of Engineering, University of Nottingham, University Park, Nottingham NG7 2RD, UK. ${ }^{5}$ Ernst Ruska-Centre for Microscopy and Spectroscopy with Electrons and Peter Grünberg Institute, Forschungszentrum Jülich, Jülich D-52425, Germany. Correspondence and requests for materials should be addressed to T.P.A. (email: t.almeida@imperial.ac.uk).
} 
M agnetic minerals in rocks record the direction and intensity of the Earth's ambient magnetic field during formation, providing, for example, information on past tectonic plate motion and the evolution of the geodynamo. In order to reliably interpret palaeomagnetic data, the mechanisms that induce and influence magnetic remanence within rocks must be fully understood. Some mechanisms such as thermoremanent magnetization are broadly understood ${ }^{1}$; however, the wide class of mechanisms that come under the heading of chemical remanent magnetization (CRM) requires more detailed study. Current models for CRM processes are, with one exception ${ }^{2}$, presently restricted to small, uniformly magnetized, singledomain (SD) grains ${ }^{3}$. Models for other types of chemical alterations are at best phenomenological ${ }^{4}$, and CRM processes in larger grains containing nonuniform magnetization states (that is, multidomain (MD) states) are little understood. Nevertheless, magnetic signals from rocks are often dominated by small MD grains that exhibit magnetic recording fidelities similar to those of SD grains (termed pseudo-SD (PSD)). For PSD grains, there are two key palaeomagnetic questions: does chemical alteration affect the directional record? To what extent does chemical alteration affect magnetization intensity?

Magnetite $\left(\mathrm{Fe}_{3} \mathrm{O}_{4}\right)$ is probably the most important magnetic mineral on Earth because of its high abundance and strong, dominating magnetization. However, its ability to preserve the remanence of the Earth's magnetic field is greatly influenced by progressive oxidation, at ambient pressures and temperatures, to less magnetic iron oxides such as maghemite $\left(\gamma-\mathrm{Fe}_{2} \mathrm{O}_{3}\right)$ or haematite $\left(\alpha-\mathrm{Fe}_{2} \mathrm{O}_{3}\right)$. Hence, oxidation of PSD $\mathrm{Fe}_{3} \mathrm{O}_{4}$ grains is a critical component of the CRM process and must be fully understood to allow for reliable interpretation of palaeomagnetic data.

Off-axis electron holography in the transmission electron microscope (TEM) allows nanometre-scale imaging of magnetic induction within and around materials as a function of applied field and temperature ${ }^{5-9}$. For example, Feinberg et al. ${ }^{10}$ used offaxis electron holography to show how the un-mixing of a titanomagnetite inclusion within a natural clinopyroxene matrix resulted in the development of an internal microstructure consisting of $\mathrm{Fe}_{3} \mathrm{O}_{4}$ prisms and ulvöspinel $\mathrm{Fe}_{2} \mathrm{TiO}_{4}$ lamellae, transforming it from an individual MD grain structure into an assemblage of magnetostatically interacting SD prisms. In particular, it was shown that the overall remanence direction was dependent on both the inclusion's elongation direction and the prism arrangements therein ${ }^{10}$, thereby demonstrating the ability of chemical alterations to affect the magnetic remanence of natural magnetic recorders.

To fully appraise the effects of chemical alterations on CRM processes, it becomes necessary to investigate changes of magnetic domain structures in grains, directly, under controlled conditions. In this context, we present the application of a range of complementary electron microscopy techniques to examine local changes in the magnetization of PSD $\mathrm{Fe}_{3} \mathrm{O}_{4}$ grains, as they chemically alter during in situ heating within a controlled oxidizing atmosphere. The technique of environmental TEM (ETEM), combined with spherical aberration $\left(\mathrm{C}_{\mathrm{S}}\right)$ correction, enables localized chemical reactions under gas atmospheres to be observed with an interpretable spatial resolution on the subångström scale ${ }^{11}$, while complementary electron energy-loss spectroscopy (EELS) investigations allow changes in material oxidation state to be appraised ${ }^{12}$. Off-axis electron holography enables nanometre-scale imaging of magnetic induction within and around magnetic grains, as a function of oxidation, through the generation of magnetic induction maps.

We show here the combined use of ETEM and off-axis electron holography to directly visualize the effect of oxidation on the recording fidelity of individual $\mathrm{PSD} \mathrm{Fe}_{3} \mathrm{O}_{4}$ grains. Construction of magnetic induction maps demonstrates a change in both strength and direction of remanent magnetic states within the $\mathrm{Fe}_{3} \mathrm{O}_{4}$ grains, as a consequence of oxidation, and we discuss how it can lead to an underestimation of the ancient geomagnetic field strength and the geodynamo magnetic moment.

\section{Results}

Effect of oxidation on strength of magnetization. Figure 1 illustrates the effect of accelerated oxidation on the magnetization of an individual, equiaxed synthetic $\mathrm{Fe}_{3} \mathrm{O}_{4}$ grain, as assessed using TEM, selected area electron diffraction (SAED) and EELS, along with associated magnetic induction maps. The bright-field TEM image of Fig. 1a shows a native, smooth-surfaced, $\sim 200 \mathrm{~nm}$ diameter $\mathrm{Fe}_{3} \mathrm{O}_{4}$ grain, as indicated by SAED (Fig. 1a, inset). EELS analysis of the $\mathrm{Fe} 2 p L_{2,3}$ edge, in the region $704-726 \mathrm{eV}$ (Fig. 1c), confirmed the assignment of pure $\mathrm{Fe}_{3} \mathrm{O}_{4}$. The $L_{2}$ edge for this sample shows the typical shape of a mixed-valence compound, that is, three visible features of differing intensities (Fig. 1c, black arrows), while the almost-shapeless $L_{3}$ edge is attributed to the combined spectral contributions of different iron sites (that is, $\mathrm{Fe}^{2+}$ at octahedral B-sites and $\mathrm{Fe}^{3+}$ at both tetrahedral $\mathrm{A}$ and octahedral B-sites), consistent with the more delocalized structure of $\mathrm{Fe}_{3} \mathrm{O}_{4}$, as compared with other mixed iron oxides ${ }^{13,14}$. The corresponding magnetic induction map of Fig. 1d exhibits evenly spaced magnetic contours, spanning from the surface to the centre of the grain, flowing in a counterclockwise direction (denoted by arrows), characteristic of a vortex state.

The bright-field TEM image of Fig. $1 \mathrm{~b}$ shows the same $\mathrm{Fe}_{3} \mathrm{O}_{4}$ grain after exposure to $9 \mathrm{mbar} \mathrm{O}_{2}$ atmosphere at $700^{\circ} \mathrm{C}$ for $8 \mathrm{~h}$ within the ETEM. Degradation of the surface of the grain is apparent, while the associated SAED pattern (Fig. 1b, inset) does not present any evidence for the formation of additional crystalline phases, with brightening of some planar reflections (for example, the -286 reflection) being attributable to slight tilting of the grain during annealing. However, the development of fine features in the associated EEL spectrum of the heated grain, taking the form of a small pre-peak in the $L_{3}$ edge and postpeak in the $L_{2}$ edge (Fig. 1c, red arrows), is indicative of a change in the $\mathrm{Fe}$ oxidation state towards $\gamma-\mathrm{Fe}_{2} \mathrm{O}_{3}$ or $\alpha-\mathrm{Fe}_{2} \mathrm{O}_{3}$ (refs $15-$ 17), as illustrated by the reference iron oxide EEL spectra displayed in Fig. 2. It is recognized that progressive oxidation induces the development of these small peaks, with complete oxidation to $\gamma-\mathrm{Fe}_{2} \mathrm{O}_{3}$ being associated with an $\sim 1.3 \mathrm{eV}$ splitting in the $L_{3}$ edge $^{15}$. Similarly, the $\mathrm{Fe} 2 p$ edge in an $\alpha-\mathrm{Fe}_{2} \mathrm{O}_{3}$ EEL spectrum is associated with a strong pre-peak located $\sim 1.6 \mathrm{eV}$ in front of the $L_{3}$ edge $^{16,17}$. Various iron hydroxides can also present similar pre-peaks in their $\mathrm{Fe} L_{3}$ edges; however, $\mathrm{O}_{2}$ is the only gas introduced into the system between acquisitions of EEL spectra, and hence the evolution of pre-peaks in this case is attributed solely to the effects of oxidation. The spacings between the central magnetic contours in the corresponding magnetic induction map (Fig. 1e), again flowing in a counterclockwise direction, were found to widen, most markedly towards the particle edge. Figure 1f,g presents magnetic contributions to the phase shifts used to construct Fig. 1d,e, respectively, and the reduction in amplitudes of the line profiles across their centres (dashed lines) is a strong indicator for loss of overall magnetic remanence in the $\mathrm{Fe}_{3} \mathrm{O}_{4}$ particle, as a consequence of oxidation (Fig. 1h, arrowed), again consistent with the progressive conversion of $\mathrm{Fe}_{3} \mathrm{O}_{4}$ towards $\gamma-\mathrm{Fe}_{2} \mathrm{O}_{3}$.

Effect of oxidation on the direction of magnetization. Figure 3 illustrates the effect of accelerated oxidation on the magnetization of an elongated $\left(\sim 250 \mathrm{~nm}\right.$ long, $\sim 150 \mathrm{~nm}$ wide) $\mathrm{Fe}_{3} \mathrm{O}_{4}$ grain. 


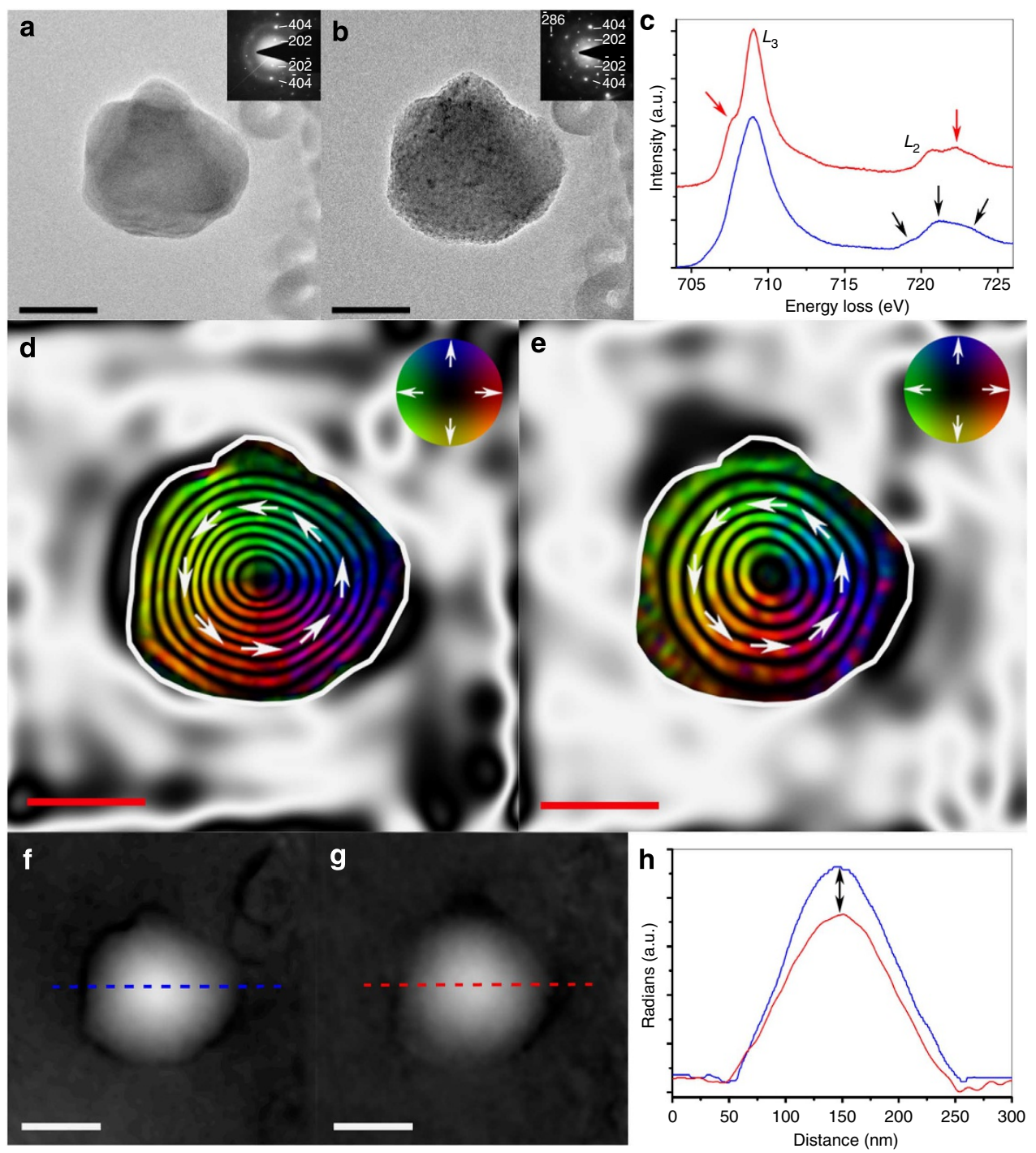

Figure 1 | Visualized effect of oxidation on the magnetization of an equiaxed $\mathbf{F e}_{\mathbf{3}} \mathbf{O}_{\mathbf{4}}$ particle. Bright-field TEM images acquired (a) before and (b) after in situ heating to $700^{\circ} \mathrm{C}$ under $9 \mathrm{mbar}$ of $\mathrm{O}_{2}$ for $8 \mathrm{~h}$ in an ETEM, with associated SAED patterns inset, indexed to $\mathrm{Fe}_{3} \mathrm{O}_{4}$ (Joint Committee on Powder Diffraction Standards (JCPDS) No. 75-449). (c) Associated EEL spectra of the Fe $2 p L_{2,3}$ edge acquired from the $\mathrm{Fe}_{3} \mathrm{O}_{4}$ particle before (blue) and after (red) annealing within the ETEM. Black arrows emphasize three differing intensities from the mixed-valence compound of $\mathrm{Fe}_{3} \mathrm{O}_{4}$, while the red arrows highlight formation of pre- and post-peaks that indicate oxidation towards $\gamma-\mathrm{Fe}_{2} \mathrm{O}_{3}$. (d,e) Magnetic induction maps determined from the magnetic contribution to the phase shift, reconstructed from holograms taken (d) before and (e) after in situ heating, revealing the vortex nature of the particle. The contour spacing is 0.79 radians for both magnetic induction maps. The magnetization direction is shown using arrows, as depicted in the colour wheel. (f, $\mathbf{g}$ ) Magnetic contributions to the phase shift, as used to reconstruct the magnetic induction maps in (d,e), respectively, and (h) line profiles across their centers before (blue) and after (red) annealing. Black arrows in $\mathbf{h}$ illustrate the loss in overall magnetic remanence. Scale bars represent $100 \mathrm{~nm}$.

The bright-field TEM image of Fig. 3a shows the grain morphology, while the associated SAED pattern (Fig. 3a, inset) indexes to $\mathrm{Fe}_{3} \mathrm{O}_{4}$, again supported by the complementary characteristic EEL spectrum of Fig. 3c. The corresponding magnetic induction map of Fig. $3 \mathrm{~d}$ reveals closely spaced magnetic contours flowing from left to right through the elongated particle, interacting with a small vortex located at the bottom, along with a component of stray magnetic field, which is indicative of a PSD state.

The bright-field TEM image of Fig. $3 \mathrm{~b}$ shows the same $\mathrm{Fe}_{3} \mathrm{O}_{4}$ grain after in situ heating in the ETEM at $700{ }^{\circ} \mathrm{C}$ under $9{\text { mbar } \mathrm{O}_{2}}^{2}$ atmosphere for $8 \mathrm{~h}$. In a similar manner to the $\mathrm{Fe}_{3} \mathrm{O}_{4}$ grain shown in Fig. 1b, the elongated particle has degraded, while brightening of various planar reflections (for example, the $-4-46$ and 44 2 reflections) in the associated SAED pattern (Fig. 3b, inset) was attributable to slight tilting of the particle during annealing. The additional development of a small pre-peak in the $L_{3}$ edge and post-peak in the $L_{2}$ edge of the corresponding EEL spectrum (Fig. 3c, red arrows) are again strong indicators for the effects of progressive oxidation towards $\gamma-\mathrm{Fe}_{2} \mathrm{O}_{3}$ or $\alpha-\mathrm{Fe}_{2} \mathrm{O}_{3}$. The associated magnetic induction map (Fig. 3e) notably exhibits two vortices with widened magnetic contour spacings, flowing in opposite directions around a central transverse axis.

\section{Discussion}

This combined ETEM and off-axis electron holography investigation has provided a visual representation of the effects of accelerated chemical oxidation on the magnetization (direction and intensity) of PSD $\mathrm{Fe}_{3} \mathrm{O}_{4}$ particles. Bright-field TEM imaging 
showed the native $\mathrm{Fe}_{3} \mathrm{O}_{4}$ particles to undergo degradation following in situ heating under an $\mathrm{O}_{2}$ atmosphere, while development of additional peaks in the Fe $2 p L_{2,3}$ edges provides

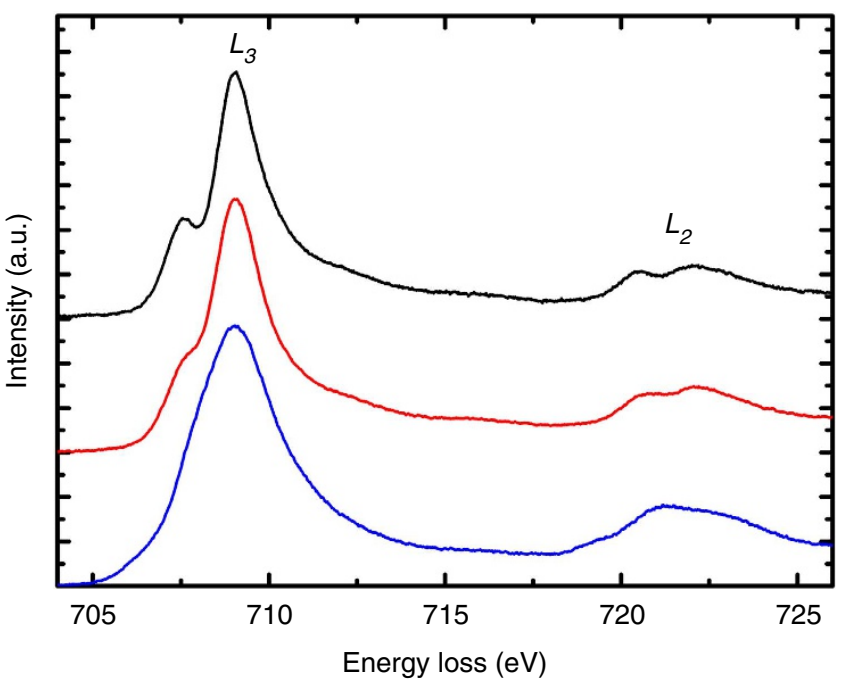

Figure 2 | EEL spectra acquired from reference iron oxide samples. EELS analysis of the $\mathrm{Fe} 2 p L_{2,3}$ edge experimentally acquired from pure samples of $\mathrm{Fe}_{3} \mathrm{O}_{4}$ (blue), $\gamma-\mathrm{Fe}_{2} \mathrm{O}_{3}$ (red) and $\alpha-\mathrm{Fe}_{2} \mathrm{O}_{3}$ (black). compelling evidence for the process of oxidation ${ }^{12}$, with the Fe $2 p$ $L_{2,3}$ edge acquired from the oxidized equiaxed $\mathrm{Fe}_{3} \mathrm{O}_{4}$ grain resembling that of pure $\gamma-\mathrm{Fe}_{2} \mathrm{O}_{3}$ (Fig. 2), as distinct from $\alpha-\mathrm{Fe}_{2} \mathrm{O}_{3}$. Intriguingly, the SAED data from the oxidized $\mathrm{Fe}_{3} \mathrm{O}_{4}$ particles provided no evidence for the development of additional crystallographic reflections. This can be expected during the bulk conversion of an inverse spinel ferrite $\mathrm{Fe}_{3} \mathrm{O}_{4}$ towards the crystallographically similar $\mathrm{Fe}^{2+}$ cation-deficient $\gamma-\mathrm{Fe}_{2} \mathrm{O}_{3}$ phase, through topotactic transformation initiated at the surface, where faint extra spots characteristic of $\gamma-\mathrm{Fe}_{2} \mathrm{O}_{3}$ would only appear in the SAED pattern along specific zone axes.

The widening of the magnetic contours around the vortex in the oxidized elongated $\mathrm{Fe}_{3} \mathrm{O}_{4}$ grain (Fig. 1e) compared with its initial state, supported by line profile differences across the magnetic contributions to the phase shift (Fig. 1h), demonstrates that chemical alteration indeed leads to a loss of magnetization intensity. Clockwise rotation of the central magnetic contours from along the major axis of the elongated $\mathrm{Fe}_{3} \mathrm{O}_{4}$ particle (Fig. 3d) to a transverse axis (Fig. 3e) demonstrates the strong effect of oxidation on the magnetization direction, an effect previously considered to be dominated by shape anistropy ${ }^{18}$. In this case, the EEL peaks characteristic of $\gamma-\mathrm{Fe}_{2} \mathrm{O}_{3}$ in the $\mathrm{Fe} 2 p L_{2,3}$ edge of the oxidized elongated grain were notably less pronounced, in comparison with the equiaxed grain, suggesting that a significant proportion of $\mathrm{Fe}_{3} \mathrm{O}_{4}$ was still present. In this case, it is proposed that the tips of elongated grains are more susceptible to oxidation because of their larger exposed surface areas and shorter diffusion pathways. Preferential tip oxidation

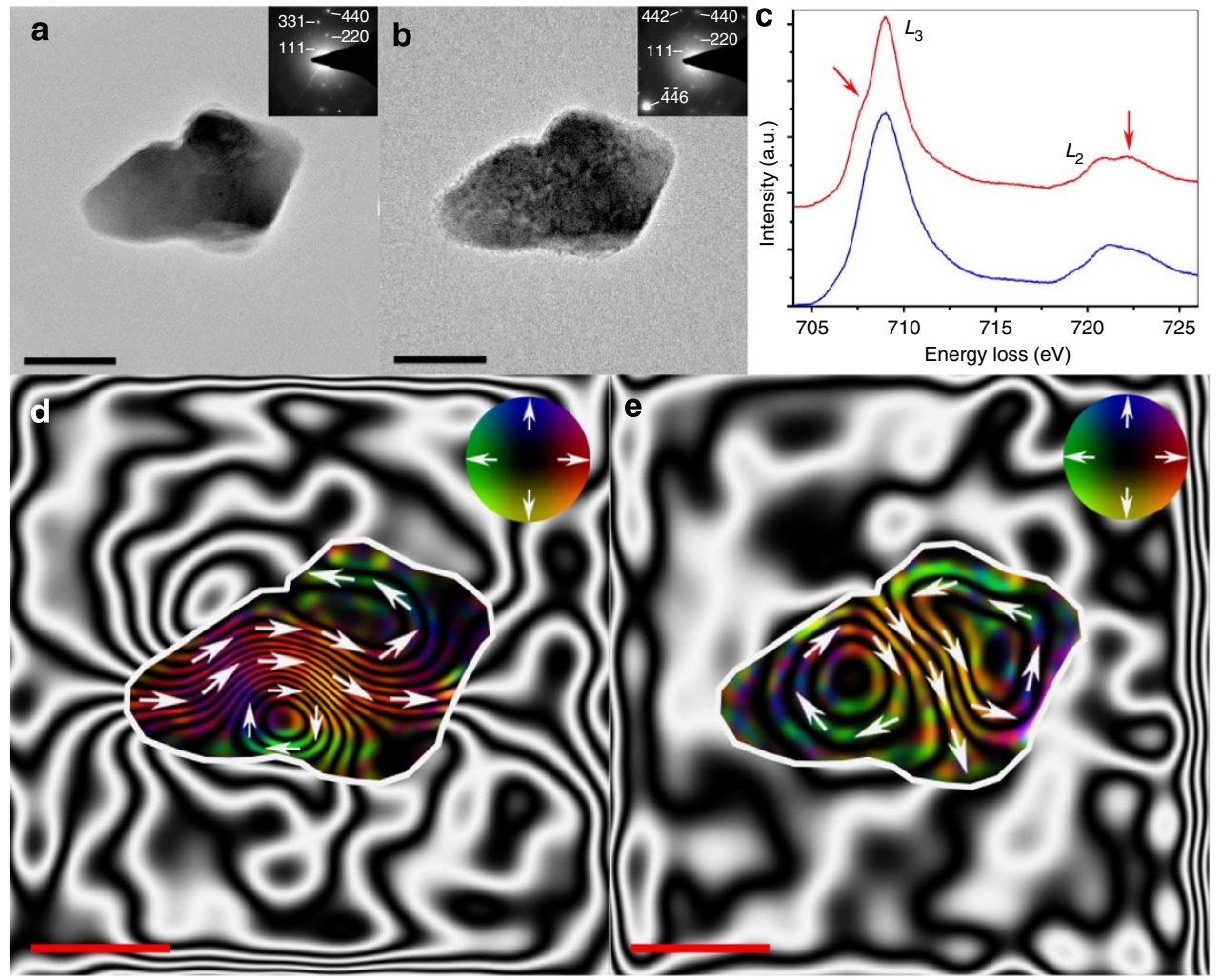

Figure 3 | Visualized effect of oxidation on the magnetization of an elongated $\mathbf{F e}_{\mathbf{3}} \mathbf{O}_{\mathbf{4}}$ particle. Bright-field TEM images acquired (a) before and (b) after in situ heating to $700{ }^{\circ} \mathrm{C}$ under $9 \mathrm{mbar}$ of $\mathrm{O}_{2}$ for $8 \mathrm{~h}$ in an ETEM, with associated SAED patterns (inset) indexed to $\mathrm{Fe}_{3} \mathrm{O}_{4}$ (JCPDS No. 75-449). (c) Associated EEL spectra of the Fe $2 p L_{2,3}$ edge acquired from the $\mathrm{Fe}_{3} \mathrm{O}_{4}$ particle before (blue) and after (red) annealing within the ETEM. Red arrows highlight the formation of pre- and post-peaks that indicate oxidation towards $\gamma-\mathrm{Fe}_{2} \mathrm{O}_{3}$. (d,e) Magnetic induction maps determined from the magnetic contribution to the phase shift reconstructed from holograms taken (d) before and (e) after in situ heating, revealing the PSD nature of the particle. The contour spacing is 0.20 radians for both magnetic induction maps. The magnetization direction is shown using arrows, as depicted in the colour wheel. Scale bars represent $100 \mathrm{~nm}$. 
towards the less magnetic $\gamma-\mathrm{Fe}_{2} \mathrm{O}_{3}$ phase would alter the overall oxide phase distribution, with chemical modification of the elongated pure $\mathrm{Fe}_{3} \mathrm{O}_{4}$ grain to a PSD grain, containing a lower aspect ratio $\mathrm{Fe}_{3} \mathrm{O}_{4}$ core, prompting a shift towards lower energy and a more favourable direction of magnetization, the in-plane component of which is represented by the magnetic induction map of Fig. 3e. Indeed, magnetic domain states exist in three dimensions, and the shift in magnetization direction between the initial and oxidized elongated $\mathrm{Fe}_{3} \mathrm{O}_{4}$ grain suggests a greater degree of complexity associated with this process than can be fully accessed by two-dimensional (2D) in-plane representations of magnetization. Comparison between experimental and simulated magnetic induction maps, derived from multiphase threedimensional (3D) micromagnetic models, could in future elucidate the $3 \mathrm{D}$ nature of the transformation observed in these magnetic domain states with chemical oxidation, providing fundamental insight into its effect on magnetic recording fidelity.

This combined ETEM and off-axis electron holography investigation has demonstrated changes in both remanent magnetic field strength and direction of magnetization within individual PSD particles as a consequence of accelerated chemical oxidation. When averaged over the millions of grains within a bulk palaeomagnetic sample, it is likely that the original directional information will be retained within the palaeomagnetic sample as not all grains will re-orientate their magnetization, although it is probable that some magnetic moments will re-align with the ambient field, giving rise to a CRM contribution to the total remanence. Certainly, the natural remanent magnetization intensity of a palaeomagnetic sample will most likely be decreased as a consequence of the oxidation process, resulting in an underestimate of ancient geomagnetic field intensity. Accordingly, palaeomagnetic data from samples showing evidence for chemical oxidation should be interpreted with care. The magnetic signal from natural rocks is inherently complex, comprising contributions from a mixture of low coercivity nonuniform MD magnetic grains, magnetically stronger nonuniform or vortex state PSD grains, and more stable high coercivity uniformly magnetized SD grains. As a step forward towards a better understanding of palaeomagnetic signals from the geomagnetic record, this study provides fundamental insight into the effect of chemical alteration on magnetic recording fidelity of the strongest magnetic mineral (magnetite) in the most commonly occurring domain state (PSD grains).

\section{Methods}

Sample details. $\mathrm{Fe}_{3} \mathrm{O}_{4}$ particles of diameter $<200 \mathrm{~nm}$ (hydrothermally synthesized by Nanostructured and Amorphous Materials, USA) were cleaned with acetone and centrifuged for $6 \mathrm{~min}$ at 6,000 r.p.m. For the purpose of ETEM investigation, the particles were dispersed in distilled water using an ultrasonic bath before deposition on an Aduro E-chip TEM sample holder (Protochips, USA).

Environmental TEM. Investigation of the in situ oxidation of $\mathrm{Fe}_{3} \mathrm{O}_{4}$ particles, under $9 \mathrm{mbar}_{2}$ atmosphere, at elevated temperature, established using a Protochips heating holder (room temperature to $700^{\circ} \mathrm{C}$ at $1{ }^{\circ} \mathrm{Cs}^{-1}$, as displayed by the Protochips temperature control), was performed using an FEI Titan E-Cell TEM with $\mathrm{C}_{\mathrm{S}}$ corrector on the objective lens, operated at $300 \mathrm{kV}$. The high temperature of $700{ }^{\circ} \mathrm{C}$ was established to help compensate for the low pressure used during ETEM, being distinct from the ambient pressure conditions found in nature. EELS analysis was performed at a spectral resolution of $\sim 0.3 \mathrm{eV}$, achieved through excitation of the monochromator, providing information on sample oxidation state. All TEM imaging and EEL spectra acquisition were performed on grains stabilized under high-vacuum conditions at ambient temperature, with in situ oxidation being performed in the absence of the electron beam to avoid any sample degradation through electron beam/material interaction during annealing.

Magnetic imaging. Off-axis electron holograms were acquired at $300 \mathrm{kV}$ using an FEI Titan 80-300 TEM in Lorentz mode, with a charge-coupled device camera and an electron biprism operated typically at $160 \mathrm{~V}$. These experiments were performed at room temperature, with an acquisition time of $4 \mathrm{~s}$. The total phase shift recorded using electron holography is sensitive to both the electrostatic potential and the inplane component of the magnetic induction in the specimen. To isolate the magnetic contribution to the phase shift, the direction of magnetization in each particle was reversed in situ in the TEM by tilting the sample $\pm 30^{\circ}$ and turning on the conventional microscope objective lens to apply a magnetic field of $2 \mathrm{~T}$ to the sample, parallel to the direction of the electron beam. The objective lens was then turned off and the sample tilted back to $0^{\circ}$ for hologram acquisition in field-free conditions (residual field $<0.2 \mathrm{mT}$ ) with the particles at remanence. Following this procedure, holograms were recorded with the particles magnetized in opposite directions, while the mean inner potential was separated from the magnetic potential, as described by Dunin-Borkowski et al. ${ }^{6}$ For the construction of magnetic induction maps, the cosine of the magnetic contribution to the phase shift was amplified to produce magnetic phase contours. Colours were added to the contours to show the direction of the projected induction, as denoted by the colour wheels.

Sample handling. In order to isolate the effects of chemical oxidation on the magnetization of the $\mathrm{Fe}_{3} \mathrm{O}_{4}$ grains, rather than possible temperature effects, samples were initially heated up to $700^{\circ} \mathrm{C}$ within the Titan 80-300 TEM for $1 \mathrm{~h}$ and then cooled under vacuum, before acquisition of off-axis electron holograms from the native $\mathrm{Fe}_{3} \mathrm{O}_{4}$ grains. The Protochips TEM holder was then transferred to the Titan ETEM for the purpose of in situ chemical oxidation, with heating at $700{ }^{\circ} \mathrm{C}$ in a 9-mbar $\mathrm{O}_{2}$ atmosphere for $8 \mathrm{~h}$, followed by imaging and EELS analysis under vacuum at ambient temperature conditions. The Protochips TEM holder and oxidized $\mathrm{Fe}_{3} \mathrm{O}_{4}$ grains were then transferred back to the Titan 80-300 TEM for acquisition of complementary off-axis electron holograms to appraise the magnetic response of the PSD grains.

\section{References}

1. Dunlop, D. J. Magnetic recording in rocks. Phys. Today 65, 31 (2012).

2. Ge, K., Williams, W., Liu, Q. \& Yu, Y. Effects of the core-shell structure on the magnetic properties of partially oxidized magnetite grains: experimental and micromagnetic investigations. Geochem. Geophys. Geosyst. 15, 2021-2038 (2014).

3. McClelland, E. Theory of CRM acquired by grain growth, and its implications for TRM acquisition and paleointensity determination in igneous rocks. Geophys. J. Inter. 126, 271-280 (1996).

4. Fabian, K. Thermochemical remanence acquisition in single-domain particle ensembles: A case for possible overestimation of the geomagnetic paleointensity. Geochem. Geophys. Geosyst. 10, Q06Z03 (2009).

5. Harrison, R., Dunin-Borkowski, R. E. \& Putnis, A. Direct imaging of nanoscale magnetic interactions in minerals. Proc. Natl Acad. Sci. USA 99, 16556-16561 (2002).

6. Dunin-Borkowski, R. E. et al. Magnetic microstructure of magnetotactic bacteria by electron holography. Science 282, 1868-1870 (1998).

7. Kasama, T., Church, N., Feinberg, J. M., Dunin-Borkowski, R. E. \& Harrison, R. J. Direct observation of ferromagnetic/ferroelastic domain interactions in magnetite below the Verwey transition. Earth Planet Sci. Lett. 297, 10-17 (2010).

8. Kasama, T. et al. Ferrimagnetic/ferroelastic domain interactions in magnetite below the Verwey transition. Part I: electron holography and Lorentz microscopy. Phase Transit. 86, 67-87 (2013).

9. Kasama, T. et al. Reversal of flux closure states in cobalt nanoparticle rings with coaxial magnetic pulses. Adv. Mater. 20, 4248-4252 (2008).

10. Feinberg, J. M. et al. Effects of internal mineral structures on the magnetic remanence of silicate-hosted titanomagnetite inclusions: an electron holography study. J. Geophys. Res. 11, B12S15 (2006).

11. Hansen, T. W., Wagner, J. B. \& Dunin-Borkowski, R. E. Aberration corrected and monochromated environmental transmission electron microscopy: challenges and prospects for materials science. Mater. Sci. Technol. 26, 1338-1344 (2010).

12. Chen, S.-Y. et al. Electron energy loss spectroscopy and $a b$ initio investigation of iron oxide nanomaterials grown by a hydrothermal process. Phys. Rev. B 79, 104103 (2009).

13. Fujii, T. et al. In situ XPS analysis of various iron oxide films grown by $\mathrm{NO}_{2}$ assisted molecular-beam epitaxy. Phys. Rev. B 59, 3195-3202 (1999).

14. Crocombette, J. P., Pollak, M., Joliet, F., Thromat, N. \& Gautier-Soyer, M. $\mathrm{X}$-ray-absorption spectroscopy at the $\mathrm{Fe} L_{2,3}$ threshold in iron oxides. Phys. Rev. B 52, 3143-3150 (1995).

15. Chen, J. et al. Magnetic circular dichroism in Fe $2 p$ resonant photoemission of magnetite. Phys. Rev. B 69, 085107 (2004).

16. van Aken, P. A. \& Lauterbach, S. Strong magnetic linear dichroism in Fe $L_{23}$ and $\mathrm{O} \mathrm{K}$ electron energy-loss near-edge spectra of antiferromagnetic hematite $\alpha-\mathrm{Fe}_{2} \mathrm{O}_{3}$. Phys. Chem. Miner. 30, 469-477 (2003).

17. Gloter, A., Douiri, A., Tence, M. \& Colliex, C. Improving energy resolution of EELS spectra: an alternative to the monochromator solution. Ultramicroscopy 96, 385-400 (2003). 
18. Tarling, D. \& Hrouda, F. Magnetic Anisotropy of Rocks 217 (Chapman and Hall, 1993).

\section{Acknowledgements}

The authors would like to thank the Natural Environment Research Council (NERC grant NE/H00534X/1) and the European Research Council for funding. We also thank the Center for Electron Nanoscopy at the Technical University of Denmark for use of their microscopy facilities

\section{Author contributions}

T.P.A. designed and carried out the experiments; A.R.M., W.W. and R.E.D.-B. conceived the study and supervised the research; T.K., T.W.H. and L.N. assisted with the experimental work and analysis; T.P.A. led the writing of the paper with contributions from A.R.M., P.D.B. and R.E.D.-B.

\section{Additional information}

Competing financial interests: The authors declare no competing financial interests.

Reprints and permission information is available online at http://npg.nature.com/ reprintsandpermissions/

How to cite this article: Almeida, T. P. et al. Visualized effect of oxidation on magnetic recording fidelity in pseudo-single-domain magnetite particles. Nat. Commun. 5:5154 doi: 10.1038/ncomms6154 (2014)

(c) (i) This work is licensed under a Creative Commons Attribution 4.0 International License. The images or other third party material in this article are included in the article's Creative Commons license, unless indicated otherwise in the credit line; if the material is not included under the Creative Commons license, users will need to obtain permission from the license holder to reproduce the material. To view a copy of this license, visit http://creativecommons.org/licenses/by/4.0/ 